\title{
Quark energy loss and shadowing in nuclear Drell-Yan process
}

\author{
Chun-Gui Duan ${ }^{1,2,5 *}$ Shu-Wen Cui ${ }^{3}$ \\ Zhan-Yuan Yan ${ }^{4}$ Guang-Lie Li ${ }^{2,5}$ \\ 1.Department of Physics, Hebei Normal University, Shijiazhuang ,050016, China \\ 2.Institute of high energy physics, The Chinese academy of sciences ,Beijing,100039,China \\ 3.Physics department,Cangzhou Teachers' College,Cangzhou,061000, China \\ 4.Department of Applied Physics,North China Electric Power University,Baoding,071003, China \\ 5.CCAST(WorldLaboratory).P.O.Box8730,Beijing,100080, China
}

\begin{abstract}
The energy loss effect in nuclear matter is another nuclear effect apart from the nuclear effects on the parton distribution as in deep inelastic scattering process. The quark energy loss can be measured best by the nuclear dependence of the high energy nuclear Drell-Yan process. By means of three kinds of quark energy loss parameterizations given in literature and the nuclear parton distribution extracted only with lepton-nucleus deep inelastic scattering experimental data, measured Drell-Yan production cross sections are analyzed for $800 \mathrm{GeV}$ proton incident on a variety of nuclear targets from FNAL E866. It is shown that our results with considering the energy loss effect are much different from these of the FNAL E866 who analysis the experimental data with the nuclear parton distribution functions obtained by using the deep inelastic lA collisions and pA nuclear Drell-Yan data . Considering the existence of energy loss effect in Drell-Yan lepton pairs production,we suggest that the extraction of nuclear parton distribution functions should not include Drell-Yan experimental data.

Keywords: Drell-Yan, energy loss, nuclear parton distribution functions
\end{abstract}

PACS:24.85.+p;13.85.QK;25.40.-h;25.75.q

Understanding the initial stages of ultrarelativistic heavy ion collisions is of

*E-mail:duancg@mail.hebtu.edu.cn 
utmost importance in order to understand the outcome of the high energy heavy ion experiments, such as the BNL relativistic heavy ion collider(RHIC) and CERN large hadron collider (LHC). Understanding the modifications of the parton distribution functions and the parton energy loss in nuclei should be the first important step towards pinning down the initial conditions of a heavy-ion collision and understanding of $J / \psi$ production which is required if it is to be used as a signal for the quark-gluon plasma in relativistic heavy ion collisions.

The high energy inelastic hadron-nucleus collisions has been studied for many decades by both the nuclear and particle physics communities ${ }^{[1]}$. By means of the nuclei, we can study the space-time development of the strong interaction during its early stages, which is inaccessible between individual hadrons. In high energy inelastic hadron-nucleus scattering, the projectile rarely retains a major fraction of its momentum after traversing the nucleus. Rather, its momentum is shared by several produced particles, which form a hadron jet in the forward direction. The classical description of this phenomena is that the projectile suffers multiple collisions and repeated energy loss in the nuclear matter. In other words, each quark or gluon in the projectile can loss a finite fraction of its energy in the nuclear target due to QCD bremsstrahlung ${ }^{[2]}$. The Drell-Yan reaction ${ }^{[3]}$ on nuclear targets provides, in particular, the possibility of probing the propagation of quark through nuclear matter, with the produced lepton pair carrying away the desired information on the projectile quark after it has travelled in the nucleus. Only initial-state interactions are important in Drell-Yan process since the dimuon in the final state does not interact strongly with the partons in the nuclei. This makes Drell-Yan scattering an ideal tool to study energy $\operatorname{loss}^{[4]}$. In addition,Drell-Yan reaction is closely related to DIS of leptons, but unlike DIS ,it is directly sensitive to anti- 
quark contributions in target parton distributions. When DIS on nuclei occurs at $x<0.08$, where $\mathrm{x}$ is the parton momentum fraction, the cross section per nucleon decreases with increasing nucleon number A due to shadowing ${ }^{[5]}$. Shadowing should also occur in Drell-Yan dimuon production at small $x_{2}$, the momentum fraction of the target parton, and theoretical calculations indicate that shadowing in the DIS and Drell-Yan reactions has a common origin ${ }^{[6]}$. Therefore,shadowing and initial state partonic energy loss are processes that occur in the proton-induced Drell-Yan reaction on nuclei.

In order to describe the modification of the initial state parton distributions in nucleus , a variety of approach to this question exist in the literature ${ }^{[7]}$. Recently,there were two trials without model dependence to obtain nuclear parton distributions from the existing world experimental data. In 1999, Eskola, Kolhinen, Ruuskanen and Salgado(EKRS ${ }^{[8]}$ suggested a set of nuclear parton distributions, which are studied within a framework of the DGLAP evolution . The measurements of $F_{2}^{A} / F_{2}^{D}$ in deep inelastic $l A$ collisions, and Drell-Yan dilepton cross sections measured in $p A$ collisions were used as constraints. The kinematical ranges are $10^{-6} \leq x \leq 1$ and $2.25 \mathrm{GeV}^{2} \leq Q^{2} \leq 10^{4} \mathrm{GeV}^{2}$ for nuclei from deuteron to heavy ones.With the nuclear parton distributions, the calculated results agreed very well with the relative EMC and Fermilab E772 experimental data ${ }^{[9]}$. In 2001, Hirai,Komano and Miyama(HKM) ${ }^{[10]}$ proposed two types of nuclear parton distributions which were obtained by quadratic and cubic type analysis, and determined by a $\chi^{2}$ global analysis of existing experimental data on nuclear structure functions without including the proton-nucleus Drell-Yan process .The kinematical ranges covered $10^{-9} \leq x \leq 1$ and $1 G e V^{2} \leq Q^{2} \leq 10^{5} \mathrm{GeV}^{2}$ for nuclei from deuteron to heavy ones . As a result, they obtained reasonable fit to the measured experimental 
data of $F_{2}$.In this work, we will use both parameterizations and investigate the nuclear dependence of the Drell-Yan process.

In order to research the partonic energy loss in nuclei, Fermilab Experiment866(E866) ${ }^{[11]}$ performed the precise measurement of the ratios of the DrellYan cross section per nucleon for an $800 \mathrm{GeV}$ proton beam incident on Be, Fe and W target at larger values of $x_{1}$, the momentum fraction of the beam parton, larger values of $x_{F}\left(\approx x_{1}-x_{2}\right)$, and smaller values of $x_{2}$ than reached by the previous experiment, Fermilab E772 ${ }^{[9]}$. The extended kinematic coverage of E866 significantly increases its sensitivity to energy loss and shadowing. This is the first experiment on the energy loss of quark passing through a cold nucleus.

After the E866 experimental data was reported,several groups have given their theoretical analysis of the data ${ }^{[12,13,14]}$. In previous report ${ }^{[14]}$, by means of EKRS and HKM nuclear parton distribution functions, we investigated the DrellYan production cross section ratios for $800 \mathrm{GeV}$ proton incident on a variety of nuclear targets in the framework of Glauber model. We found that the theoretical results with energy loss are in good agreement with the Fermilab E866 experiment by means of HKM nuclear parton distributions. However, the calculated results without energy loss can give good fits by using EKRS nuclear parton distribution functions. In this letter, the nuclear dependence of the pA Drell-Yan production cross sections are studied by combining the quark energy loss parametrization ${ }^{[15,18,19]}$ given in literature with the EKRS and HKM nuclear parton distribution.

In the Drell-Yan process, the leading-order contribution is quark-antiquark annihilation into a lepton pair. The annihilation cross section can be obtained from the $e^{+} e^{-} \rightarrow \mu^{+} \mu^{-}$cross section by including the color factor $\frac{1}{3}$ with the 
charge $e_{f}^{2}$ for the quark of flavor $f$.

$$
\frac{d \hat{\sigma}}{d M}=\frac{8 \pi \alpha^{2}}{9 M} e_{f}^{2} \delta\left(\hat{s}-M^{2}\right)
$$

where $\sqrt{\hat{s}}=\left(x_{1} x_{2} s\right)^{1 / 2}$, is the center of mass system (c.m.system) energy of $q \bar{q}$ collision,$x_{1}\left(\operatorname{resp} . x_{2}\right)$ is the momentum fraction carried by the projectile (resp.target) parton, $\sqrt{s}$ is the center of mass energy of the hadronic collision, and $M$ is the invariant mass of the produced dimuon. The hadronic Drell-Yan differential cross section is then obtained from the convolution of the above partonic cross section with the quark distributions in the beam and in the target :

$$
\frac{d^{2} \sigma}{d x_{1} d M}=K \frac{8 \pi \alpha^{2}}{9 M} \frac{1}{x_{1} s} \sum_{f} e_{f}^{2}\left[q_{f}^{p}\left(x_{1}\right) \bar{q}_{f}^{A}\left(x_{2}\right)+\bar{q}_{f}^{p}\left(x_{1}\right) q_{f}^{A}\left(x_{2}\right)\right]
$$

where $K$ is the high-order QCD correction, $\alpha$ is the fine-structure constant, the sum is carried out over the light flavor $f=u, d, s$, and $q_{f}^{p(A)}(x)$ and $\bar{q}_{f}^{p(A)}(x)$ are the quark and anti-quark distributions in the proton (nucleon in the nucleus A). In order to obtain the $x_{1}$ dependence of Drell-Yan production, we shall deal in the following with the single differential cross section,

$$
\frac{d \sigma}{d x_{1}}=K \frac{8 \pi \alpha^{2}}{9 x_{1} s} \sum_{f} e_{f}^{2} \int \frac{d M}{M}\left[q_{f}^{p}\left(x_{1}\right) \bar{q}_{f}^{A}\left(x_{2}\right)+\bar{q}_{f}^{p}\left(x_{1}\right) q_{f}^{A}\left(x_{2}\right)\right]
$$

where the integration over the dimuon mass is performed in the range given from E866 experiment.

Now let us take into account of the energy loss of the fast quarks moving through the cold nuclei.There were three expressions for the average change in incident parton momentum fraction in literature. The first was given by Gavin and Milana $^{[15]}$ in 1992.They analyzed the depletion at high Feynman $\mathrm{x}$ in large relative to small nuclear targets from measurements of the Drell-Yan process and of charmonium and bottomonium production at CERN ${ }^{[17]}$ and Fermilab E772 ${ }^{[9,16]}$. 
They proposed that energy loss due to multiple parton scattering can consistently explain the high- $x_{F}$ depletion in the Drell-Yan process and $J / \psi$ production. The following expression was assumed for the average change in incident parton momentum fraction :

$$
\Delta x_{1}=\kappa_{1} x_{1} A^{1 / 3},
$$

where the factor $\kappa_{1}$ may have a $Q^{2}$ dependence.From comparison to the experimental data and neglecting shadowing, an upper bound on $d E / d z$-the incident quark energy loss per unit length — was obtained,the value is $1.5 \mathrm{GeV} / \mathrm{fm}$. This result was questioned by Brodsky and Hoyer ${ }^{[18]}$, who argued that the time scale for gluon bremsstrahlung need to be taken into account.By analogy with the photon bremsstrahlung process, they derived the quantum mechanical bound on the amount of radiative energy loss suffered by high energy quarks and gluons in nuclear matter,

$$
\Delta x_{1} \approx \frac{\kappa_{2}}{s} A^{1 / 3},
$$

where $s$ denotes the square of the proton-nucleus center of mass energy. The formulation developed by Brodsky and Hoyer was extended by Baier et al. ${ }^{[19]}$ They studied the medium-induced $p_{\perp}$-broadening and induced gluon radiation spectrum of a high energy quark or gluon traversing a large nucleus.Multiple scattering of the high energy parton in nuclei was treated in the Glauber approximation. Baier et al. found that the partonic energy loss depended on a characteristic length and the broadening of the squared transverse momentum of the parton. For finite nuclei,both factors vary as $A^{1 / 3}$, so they predicted

$$
\Delta x_{1} \approx \frac{\kappa_{3}}{s} A^{2 / 3} .
$$

Using these energy loss expressions, we can obtain empirical values for the $\kappa$ 's by 
fitting the FNAL E866 Drell-Yan cross section ratios versus the incident proton's momentum fraction and dimuon effective mass.

If considering the quark energy loss in nuclei, the incident quark momentum fraction can be shifted from $x_{1}^{\prime}=x_{1}+\Delta x_{1}$ to $x_{1}$ at the point of fusion. Combining the shadowing with initial state energy loss, the production cross section in pA Drell-Yan process can be written as

$$
\frac{d \sigma}{d x_{1}}=K \frac{8 \pi \alpha^{2}}{9 x_{1} s} \sum_{f} e_{f}^{2} \int \frac{d M}{M}\left[q_{f}^{p}\left(x_{1}^{\prime}\right) \bar{q}_{f}^{A}\left(x_{2}\right)+\bar{q}_{f}^{p}\left(x_{1}^{\prime}\right) q_{f}^{A}\left(x_{2}\right)\right] .
$$

In order to pin down quark energy loss by comparing with the experimental data from E866 collaboration ${ }^{[11]}$,we introduce the nuclear Drell-Yan ratios as:

$$
R_{A_{1} / A_{2}}\left(x_{1}\right)=\frac{d \sigma^{p-A_{1}}}{d x_{1}} / \frac{d \sigma^{p-A_{2}}}{d x_{1}} .
$$

The integral range on $\mathrm{M}$ is determined according to the E866 experimental kinematic region.In our theoretical analysis,$\chi^{2}$ is calculated with the Drell-Yan differential cross section rations $R_{A_{1} / A_{2}}$ as

$$
\chi^{2}=\sum_{j} \frac{\left(R_{A_{1} / A_{2}, j}^{\text {data }}-R_{A_{1} / A_{2}, j}^{\text {theo }}\right)^{2}}{\left(R_{A_{1} / A_{2}, j}^{\text {err }}\right)^{2}},
$$

where the experimental error is given by systematic errors as $R_{A_{1} / A_{2}, j}^{e r r}$, and $R_{A_{1} / A_{2}, j}^{d a t a}($ $R_{A_{1} / A_{2}, j}^{\text {theo }}$ ) indicates the experimental data (theoretical values ) for the ratio $R_{A_{1} / A_{2}}$.

Taking advantage of the EKRS ${ }^{[8]}$ nuclear parton distribution functions and $\kappa_{1}=0, \kappa_{2}=0, \kappa_{3}=0$ (without energy loss effects), the obtained $\chi^{2}$ value is $\chi^{2}=51.4$ for the 56 total data points. The $\chi^{2}$ per degrees of freedom is given by $\chi^{2} /$ d.o.f. $=0.918$. It is apparent that theoretical results without energy loss effects agree very well with the E866 experimental data, which results from EKRS parametrization of nuclear parton distributions studied with including the E772 Drell-Yan data ${ }^{[16]}$. Because of employing the EKRS global phenomenological analysis, the E866 analysis missed the energy loss effect of quarks in nuclei. 
In what follows, we consider also combining HKM cubic type of nuclear parton distribution ${ }^{[10]}$ with the three quark energy loss parameterizations. With $\kappa_{1}=$ $0, \kappa_{2}=0, \kappa_{3}=0$ (without energy loss effects), the obtained $\chi^{2}$ per degrees of freedom is $\chi^{2} /$ d.o.f. $=2.516$. With $\kappa_{1}=0.0036, \kappa_{2}=3.45, \kappa_{3}=0.46$ (with energy loss effects), the obtained $\chi^{2}$ per degrees of freedom are $\chi^{2} /$ d.o.f. $=1.00048,1.022,1.053$, respectively. The results given by HKM quadratic type are nearly the same as these above. As an example,the calculated results with energy loss expression Eq.(5)are shown in Fig.1 and Fig.2. which is the Drell-Yan cross section ratios for Fe to Be and $\mathrm{W}$ to Be as functions of $x_{1}$ for various interval of $M$,respectively.The solid curves are the ratios with only the nuclear effect on the parton distribution as in DIS scattering process, and the dotted curves correspond to an energy loss effect: $\kappa_{2}=3.45 \mathrm{GeV}^{2}$ with nuclear effect on structure function .From comparison with the experimental data, it is found that our theoretical results with energy loss effect are in good agreement with the Fermilab E866. However,the values of $\kappa_{1}, \kappa_{2}, \kappa_{3}$ are very different from those by the E866 Collaboration ${ }^{[1]}$, which results from employing EKRS nuclear parton distributions. The EKRS analysis itself included the DrellYan data from E772, with the presumption that the low- $x_{2}$ nuclear dependence arose entirely from shadowing. This clearly introduced an inconsistency into the E866 search for energy loss. Therefore,we can conclude that the extraction of nuclear parton distribution functions should not include Drell-Yan experimental data because of the energy loss of the incoming partons.

In Eq.(4), the average loss of projectile parton vary with the variable $x_{1}$. It is difficult to obtain a constant energy loss $d E / d z(G e V / f m) . \kappa_{3}=0.46$ shows us that the observed energy loss of the incident quarks is $\Delta E<0.21 \mathrm{GeV} / \mathrm{fm}^{2} \times$ $L^{2} . \kappa_{2}=3.45$ in Eq.(5)indicates that the incident quarks lose energy with a con- 
stant rate $d E / d z=2.04 G e V / f m$ if $\left\langle L>=3 / 4\left(1.2 A^{1 / 3}\right) f m\right.$ is used as the average path length. The value of $d E / d z$ is not obviously consistent with that given by M.B.Johnson et al. ${ }^{[12]}$, which is $-d E / d x=3.14 \pm 0.53 \mathrm{GeV} / \mathrm{fm}$.M.B.Johnson et al. examined the effect of initial state energy loss on the Drell-Yan cross section ratios from E866 by employing a new formulation of the Drell-Yan process in the rest frame of nucleus.It is obvious that an intuitive explanation of the quark energy loss depends on the reference frame of the target nucleus. It is well-known that the physical observables can not depend on our choice of reference frame. Therefore,further theoretical and experimental studies are needed to determine the value of incident quark energy loss per unit length,and verify whether the energy loss is linear or quadratic with the path length.

With considering the existence of energy loss effect in Drell-Yan lepton pairs production,we suggest that the extraction of nuclear parton distribution functions should not employ pA Drell-Yan experimental data.Although there are currently abundant data on electron and moun deep inelastic scattering, it is difficult to determine valence quark distributions in the small $\mathrm{x}$ region and the anti-quark distributions .Only valence quark distributions in medium x region can be relatively well determined. It is well considered that the precise nuclear parton distributions must be known in order to calculate cross sections of high energy nuclear reactions accurately and find a signature of quark-gluon plasma in high energy heavy-ion reactions.We suggest using precise neutrino scattering experimental data, which can provide a good method for measuring the $F_{2}\left(x, Q^{2}\right)$ and $x F_{3}\left(x, Q^{2}\right)$ structure functions. Using the average of $x F_{3}^{\nu A}\left(x, Q^{2}\right)$ and $x F_{3}^{\bar{\nu} A}\left(x, Q^{2}\right)$, the valence quark distribution functions can be well pinned down ${ }^{[20]}$. Combining the lepton inelastic scattering data with the neutrino scattering experiments, valence quark and anti- 
quark distribution functions will be obtained in the future, which makes us good understanding the energy loss effect in high energy nuclear collisions.

In summary, we have made a leading-order analysis of E866 data in nuclei by taking into account of the energy loss effect of fast quarks . Our theoretical results with quark energy loss are in good agreement with the Fermilab E866 experiment by means of the parametrization of nuclear parton distributions studied without nuclear Drell-Yan process, which is the same as that in our previous work ${ }^{[14]}$.we suggest that the extraction of nuclear parton distribution functions should not employ pA Drell-Yan experimental data.In order to determine the amount of energy loss and the appropriate path length,we desire to operate precise measurements of the nuclear dependent of Drell-Yan production at the Fermilab Main Injector(FMI,120GeV proton beam ${ }^{[21]}$ and the Japan Hadron Facility(JHF,50GeV pro-

ton beam ${ }^{[22]}$, where shadowing effect disappears and energy loss effect of fast quarks could provide the dominant nuclear dependence.

Acknowledgement:This work is partially supported by Natural Science Foundation of China(90103020,10075057,10175074), CAS Knowledge Innovation Project (KJCX2-SW-N02),Major State Basic Research Development Program (G20000774), Natural Science Foundation of Hebei Province(103143)

\section{References}

[1] W.Busza R Ledoux, Ann.Rev.Nucl.Part.Sci.,38(1989)119.

[2] D.S.Barton et al.,Phys.Rev.D27(1983)2580.

[3] S.Drell and T.M.Yan, Phys.Rev,Lett.,25(1970)316.

[4] G.T.Garvey and J.C.Peng ,Phys.Rev.Lett.,90(2003)092302. 
[5] P.Amaudruz et al.,Z.Phys.C51(1991)387;57(1993)211.

[6] S.J.Brodsky,A.Hebecker,E.Quark.Phys.Rev.D.55.(1997)2584.

B.Kopelievich.Proc.workshop Hirschegg 1995, ed.by H.Feldmeier and W.Norenberg,Darmstadt,1995,102.

[7] M.Arneodo.et.al.(EMC), Nucl.Phys,B441(1995)3.

D.F.Geesaman,K.Saito and A.W.Thomas,Ann.Rev.Nucl.Part.Sci.45(1995)337

[8] K.J.Eskola,V.J.Kolinen and C.A.Salgado(EKS),Eur.Phys.J.C9(1999)61.

K.J.Eskola,V.J.Kolinen and P.V.Ruuskanen,Nucl.Phys.B535(1998)351.

[9] D.M.Adle et al.(E772),Phys.Rev.Lett.,64(1990)2479

[10] M.Hirai,S.Kumano,M.Miyama(HKM) ,Phys.Rev.D64(2001)034003.

[11] M.A.Vasiliev,et.al.(E866),Phys.Rev.Lett.83(1999)2304.

[12] M.B.Johnson et al.,Phys.Rev.,C65,025203(2002).

[13] Francois Arleo,Phys.Lett.B532,231(2002).

[14] C.G.Duan L.H.Song,L.J.Huo and G.L.Li,Eur.Phys.J.C29,557(2003)

Duan Chungui,WANG Hong-MinLI Guang-Lie , Chin.Phys.Lett., $19,485(2002)$.

[15] S.Gavin and J.Milana,Phys.Rev.lett.,68(1992)1834.

[16] D.Alde et al.,Phys.Rev.Lett.,66(1991)133.

[17] J.Badier et al., Z.Phys.c20(1983)101.

[18] S.J.Brodsky and P.Hoyer,Phys.lett.,B298,165(1993). 
[19] R.Baier et al.,Nucl.Phys.B484(1997)265 hep-ph/9804212.

[20] M.Botje,Eur.Phys.J.C14(2000)285 hep-ph/9912439.

[21] D.Geesaman et. al.,Fermolab Proposal No.E906,1999.

[22] J.C.Peng et. al. hep-ph/0007341;M.Asakawa et. al.,KEK Report No 2000-11.

Figure caption

Fig.1 The nuclear Drell-Yan cross section ratios $R_{A_{1} / A_{2}}\left(x_{1}\right)$ on Fe to Be for various intervals M. Solid curves correspond to nuclear effect on structure function . Dotted curves show the combination of shadowing and energy loss effect with HKM cubic type of nuclear parton distributions. The experimental data are taken from the E866[11].

Fig.2 The nuclear Drell-Yan cross section ratios $R_{A_{1} / A_{2}}\left(x_{1}\right)$ on $\mathrm{W}$ to Be for various intervals M. The comments is the same as Fig.1 

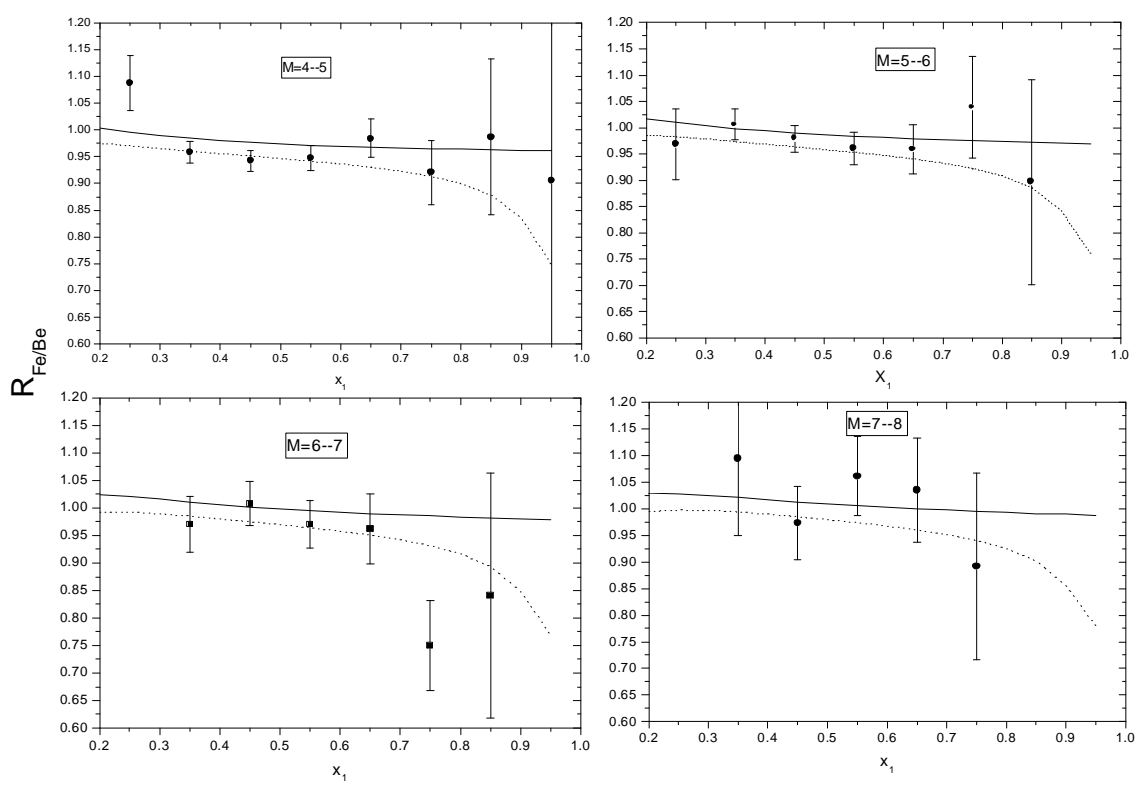

Fig.1

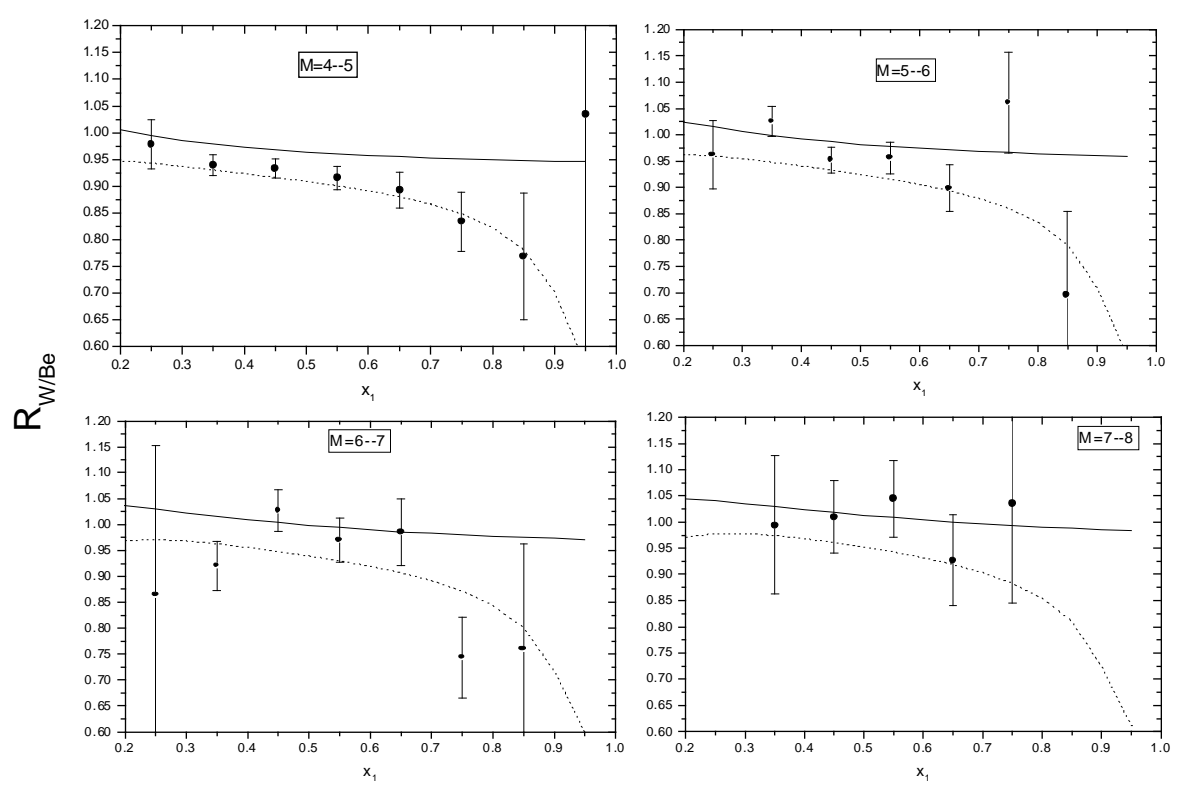

Fig.2 